\title{
Utilización de la parathormona como predictor de hipoparatiroidismo postiroidectomía total
}

\author{
Use of parathormone as a predictor of hypoparathyroidism after total thyroidectomy
}

\author{
Alejandro Yanzón-de la Torre, Natalia L. Gómez*, Carla Abuawad y Marcelo F. Figari \\ Sector Cirugía de Cabeza y Cuello, Servicio de Cirugía General, Hospital Italiano de Buenos Aires, Buenos Aires, Argentina
}

\begin{abstract}
Resumen
Antecedentes: El hipoparatiroidismo posoperatorio constituye la complicación más frecuente de la tiroidectomía total. Se asocia, entre otras cosas, a internación prolongada y múltiples pruebas de laboratorio, y con ello a un incremento en los costos de salud. La identificación de pacientes con mayor riesgo de padecer esta complicación permite realizar un tratamiento precoz, disminuyendo el costo económico y evitando complicaciones asociadas a un retraso en la externación. Se han descrito diversos predictores para identificar tempranamente a los pacientes en riesgo; en los últimos años, ha tomado particular relevancia el uso de la parathormona (PTH). Objetivo: El objetivo del presente trabajo es revisar la literatura existente sobre la utilidad de la PTH como predictor de hipocalcemia postiroidectomía. Método: Se realizó una búsqueda en PubMed revisando la evidencia existente sobre eficacia de la PTH como predictor de hipocalcemia posoperatoria, su repercusión económica, el tiempo óptimo para la toma de muestra y el modo de implementación. Conclusión: El uso de la PTH permite predecir con adecuada sensibilidad, especificidad, valor predictivo negativo y valor predictivo positivo los pacientes en riesgo de padecer hipocalcemia posoperatoria. Sin embargo, los valores de corte, los tiempos de toma de muestra y la cantidad de estas varían entre los autores, por lo que persisten algunos interrogantes acerca de la estandarización de su uso.
\end{abstract}

Palabras Clave: Hipocalcemia. Hipoparatiroidismo. Hormona paratiroidea. Iridectomía.

\begin{abstract}
Background: Post-operative hypoparathyroidism is the most frequent complication after total thyroidectomy. It represents one of the main causes of prolonged hospital-stay and is associated with a significant increase in health costs. The identification of patients with higher risk of suffering this complication allows early treatment, reduces clinical complications and adequate the use of health resources. Throughout history, several predictors have been used to stratify patients at risk. In recent years the use of parathormone parathyroid hormone (PTH) has taken particular interest. Objective: To review the existing literature on the use of PTH as a predictor of hypocalcemia after thyroidectomy. Method: A medline search was performed. We reviewed the existing evidence on efficacy of PTH as a predictor of post-operative hypocalcemia, economic impact, optimal time for sampling and implementation mode. Conclusion: The use of PTH predicts with adequate sensitivity, specificity, negative and positive predictive value the risk for the patients to suffer post-operative hypocalcemia. Cut-off values and sampling number and time vary among authors; as a result, more data is needed to reach a conclusion about the standardization of use after a total thyroidectomy procedure. It use could be beneficial not only for patients but also for care providers as health cost might be diminished.
\end{abstract}

Key Words: Hypocalcemia. Hypoparathyroidism. Parathyroid hormone. Thyroidectomy.

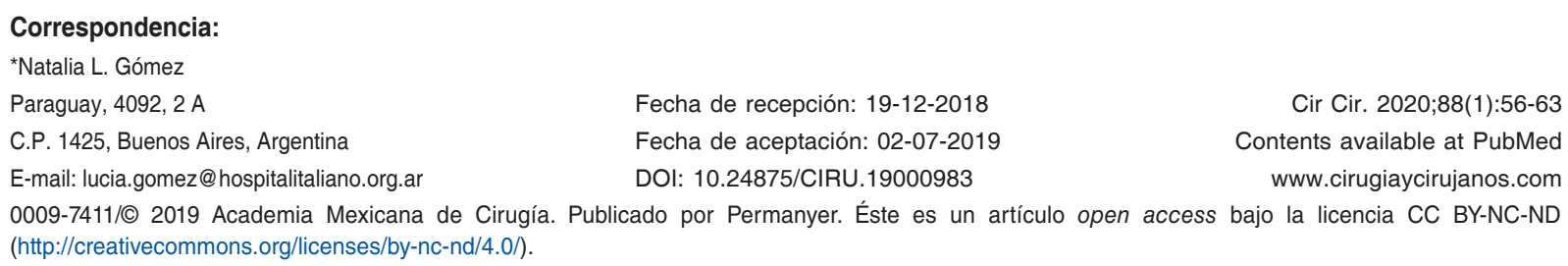




\section{Introducción}

La homeostasis del calcio es de crítica importancia para la fisiología humana. La hipocalcemia puede ocasionar desde parestesias leves hasta complicaciones con riesgo de vida, como cuadros confusionales graves, tetania 0 arritmias graves ${ }^{1-3}$. Durante la cirugía tiroidea, como consecuencia de la desvascularización o remoción de las glándulas paratiroideas, puede producirse un descenso de las concentraciones séricas de parathormona (PTH), ocasionando hipoparatiroidismo transitorio o persistente ${ }^{4}$. Este fenómeno, junto con otros factores, provoca una disminución de las concentraciones séricas de calcio en el posoperatorio ${ }^{5}$.

El hipoparatiroidismo posoperatorio representa la complicación más frecuente luego de una tiroidectomía total ${ }^{6,7}$. Se clasifica como transitorio cuando su duración es menor de 6 meses y como definitivo cuando persiste por más tiempo. El hipoparatiroidismo transitorio se presenta en el 1,7-68\% de los pacientes, mientras que su forma definitiva afecta al $1-12 \%$ en las distintas series publicadas ${ }^{4,8-11}$. Esta complicación se manifiesta en las primeras 24-96 horas posteriores a la cirugía, motivo por el cual en muchas instituciones los pacientes permanecen internados durante este tiempo ${ }^{4,7,8}$. Una vez instalado el hipoparatiroidismo, requiere tratamiento con reposición de calcio y vitamina $D, y$ un monitoreo estricto con el fin de evitar, detectar o tratar las manifestaciones graves de la hipocalcemia (arritmias, tetania, etc.) ${ }^{2}$.

La estratificación de los pacientes según el riesgo de padecer hipocalcemia permite instaurar un tratamiento efectivo en aquellos categorizados como de alto riesgo, y posibilita una rápida externación para aquellos de bajo riesgo, lo que se traduce en menores costos hospitalarios ${ }^{7,10,12}$. El dosaje seriado del calcio sérico ha constituido el predictor bioquímico más utilizado para monitorizar la función paratiroidea posoperatoria, debido sobre todo a su relativa accesibili$\mathrm{dad}^{7,8,13}$. No obstante, presenta múltiples limitaciones, como el costo que representa analizar varias muestras de sangre, las molestias o las potenciales complicaciones derivadas de repetidas punciones, así como también que los valores normales de la calcemia dentro de las primeras 24 horas no parecen predecir adecuadamente el comportamiento del calcio sérico en las primeras horas del posoperatorio ${ }^{4,7-9}$. Esto se debería a que la meseta o plateau de la calcemia posquirúrgica se alcanza entre las 48 y las 72 horas tras la cirugía 4 . Esta relativa baja sensibilidad para predecir la hipocalcemia posoperatoria (una muestra a las $24 \mathrm{~h}$ de la cirugía tendría una sensibilidad del $19-91 \%)^{7,8}$ justifica que algunos centros prolonguen la estadía hospitalaria o mediquen de manera preventiva y sistemática a sus pacientes, aun con los perjuicios que esto conlleva $a^{3,7,9,10,14}$.

Existen otros predictores que intentan identificar a los pacientes con riesgo de hipoparatiroidismo; entre ellos, la concentración sérica de vitamina $D$ preoperatoria, el dosaje de fosfatemia, la calcitonina y la magnesemia posoperatorias, el número de glándulas identificadas en la cirugía, la necesidad de autoimplante paratiroideo, etc. ${ }^{8}$. Sin embargo, el dosaje posoperatorio de PTH es el predictor que mayor auge ha tomado en los últimos años y que genera numerosos debates en cuanto a su sistemática de implementación durante la internación, variables de metabolización y excreción de la hormona, costos, etc.?

El objetivo de este trabajo es hacer una revisión no sistemática de la literatura publicada en PubMed, entre 1998 y 2017, acerca de la PTH como predictor de hipocalcemia en el posoperatorio inmediato y mediato de una tiroidectomía total.

\section{Método}

Se realizó una búsqueda en PubMed de textos en inglés utilizando los siguientes términos $\mathrm{MeSH}$ y los operadores booleanos clave: parathormona AND/OR hipocalcemia AND/OR tiroidectomía AND/OR posoperatoria. Se incluyeron aquellos trabajos que describían la eficacia de la PTH como predictor de hipocalcemia posoperatoria, factores económicos asociados a su uso, tiempo óptimo para la toma de muestra y modo de implementación. Se limitó la búsqueda a artículos publicados entre 1998 y 2019.

\section{Eficacia de la PTH como predictor}

La PTH es un péptido de 84 aminoácidos sintetizado en las glándulas paratiroideas. Su secreción depende de la concentración sérica de calcio iónico: a menor calcemia, mayor secreción de PTH. Eleva las concentraciones séricas de calcio a través de distintos mecanismos. Tiene una vida media corta, de $1 \mathrm{a}$ 4 minutos, con un rápido metabolismo hepático y eliminación renal².

Numerosos estudios han utilizado la PTH como predictor de hipocalcemia postiroidectomía, demostrando valores adecuados de sensibilidad, especificidad y 
valores predictivos negativo y positivo, con una ligera superioridad con respecto al monitoreo del calcio,8,10,13. Si bien los estudios son muy heterogéneos en cuanto a variables y formas de medición, la sensibilidad propuesta varía del 69 al $100 \%$ en una muestra tomada entre la primera y las 24 horas del posoperatorio $0^{7,8,15}$.

Esta evidencia permitiría aseverar que valores normales de PTH intraoperatorios o posoperatorios excluyen la posibilidad de presentar una hipocalcemia significativa ${ }^{10,14}$. Aunque de manera poco frecuente, los pacientes con valores normales de PTH intraoperatoria podrían presentar hipocalcemia, pero mínima ${ }^{16,17}$. Contrariamente, bajos valores de PTH posoperatoria se asocian con un elevado índice de hipocalcemia, por lo que un seguimiento más estricto y una eventual medicación serían recomendables ${ }^{16}$. Uno de los trabajos más recientes es el de Calvo Espino, et al. ${ }^{18}$, quienes demuestran que la medición de la PTH en el primer día de posoperatorio con un resultado $<5 \mathrm{pg} / \mathrm{ml}$ tiene una sensibilidad del 95\% y una especificidad del $77 \%$ para predecir hipoparatiroidismo permanente; asimismo, el dosaje del calcio sérico presentó una sensibilidad un poco menor (92\%) y una especificidad aún más baja (65\%). Almquist, et al..$^{19}$ presentan similares conclusiones, ya que establecen que valores muy bajos de PTH posoperatoria tienen un elevado riesgo de hipoparatiroidismo definitivo, por lo que valores normales de PTH posoperatoria permitirían excluir la posibilidad de presentarlo. En igual sintonía, manifiestan que el monitoreo de la calcemia en el posoperatorio, por sus variables, no permite predecir adecuadamente el riesgo de hipoparatiroidismo definitivo (duración $>6$ meses). Por su parte, Hermann, et al. ${ }^{20}$ determinaron que unos valores de PTH iguales o por debajo de $6 \mathrm{pg} / \mathrm{ml}$ tendrían una sensibilidad del $100 \%$ y un valor predictivo positivo del $15 \%$ para el hipoparatiroidismo definiti$\mathrm{vo}^{20}$. Sin embargo, otros estudios, como el de Sahli, et al. ${ }^{15}$, descartan dicha correspondencia. Su trabajo, con 218 pacientes incluidos, descartó la PTH medida dentro de la primera hora poscirugía como predictor confiable de hipocalcemia, alegando su baja sensibilidad (36,5\%); entre sus conclusiones, destaca la necesidad de estudios aleatorizados para llegar a una conclusión más acertada.

\section{Repercusión económica}

La repercusión en los costos de salud que genera el dosaje de la PTH tiene variaciones entre cada centro y país, pero como generalidad el costo absoluto suele ser mayor que un análisis de calcemia ${ }^{21}$. A pesar de esto, el monitoreo de la PTH se asocia con una disminución en los costos totales $7,8,10,12,13,21,22$. Esto se debe a que, dado que la curva de descenso de la PTH alcanza su meseta dentro de los primeros minutos del posoperatorio, o para muchos antes de finalizada la cirugía, un único análisis sería suficiente ${ }^{4}$. En cambio, el dosaje de la calcemia requiere numerosas extracciones.

Otro punto a considerar en la disminución de los costos totales es que, al poder obtenerse una sola muestra (en el posoperatorio inmediato o muy cercano a la finalización de la cirugía), el alta puede ser otorgada en forma precoz, acortando la estadía hospitalaria y ahorrando en medicación innecesaria ${ }^{7,22}$. Es importante destacar que, si bien la exactitud de la PTH como predictor no es del $100 \%$, la hipocalcemia es muy infrecuente con valores normales de PTH perioperatoria. Siguiendo este concepto, un solo valor normal de PTH posoperatoria permitiría el alta temprana, incluso en el mismo día de la cirugía ${ }^{10,22,23}$. En concordancia con lo expuesto, Payne, et al..$^{12}$ demostraron en su serie un ahorro de US\$ 760 por paciente cuando compararon el monitoreo de la PTH con la calcemia seriada, explicado por el acortamiento de la internación.

\section{Tiempo óptimo para la toma de la muestra y modo de implementación}

A pesar de la claras ventajas que la PTH ofrece como predictora de hipocalcemia posoperatoria, aún existen controversias en relación con el momento óptimo para la toma de la muestra y con la logística de implementación (Tabla 1).

Quizás el mayor interrogante sea el momento para realizar el dosaje. Diversos autores proponen la toma de la muestra durante la cirugía o al cierre de la piel; otros, un análisis a lo largo de las primeras 24 horas, pasando por 1, 4, 6 y 8 horas del posoperatorio; y otros proponen tomarla directamente durante el primer día posoperatorio',7,9,12,14-16,18,22,24-27.

Como se mencionó previamente, hay grupos que proponen tomar la muestra en el intraoperatorio o posoperatorio inmediato con el paciente aún bajo anestesia. Quiros, et al. ${ }^{1}$ establecen una relación entre valores de $\mathrm{PTH}<10 \mathrm{pg} / \mathrm{ml}$ al momento del cierre de la piel con el hipoparatiroidismo posoperatorio y la hipocalcemia, por lo que sugieren realizar una reposición temprana de vitamina $D$ en los pacientes que presenten estos valores. En concordancia, Lang, 
Tabla 1. Resumen de estudios con sus diseños y principales hallazgos y conclusiones

\begin{tabular}{|c|c|c|c|c|c|c|c|c|}
\hline Autores & Año & Diseño & $\mathrm{n}$ & PTH & $\begin{array}{l}\text { Tiempo } \\
\text { para PTH }\end{array}$ & Control & $\begin{array}{c}\text { Valor de corte } \\
\text { PTH }\end{array}$ & Conclusión \\
\hline Quiros, et al. ${ }^{1}$ & 2005 & Prospectivo & 72 & PTHi & $\begin{array}{l}\text { Cierre; } \\
\text { mes } 1 \text { pos }\end{array}$ & $\begin{array}{l}\text { Calcemia } \\
\text { al mes }\end{array}$ & $<10 \mathrm{pg} / \mathrm{ml}$ & $\begin{array}{l}\text { Una sola medición de PTHi } \\
\text { al cierre de piel predice } \\
\text { hipoparatiroidismo }\end{array}$ \\
\hline Lang, et al. ${ }^{3}$ & 2012 & Prospectivo & 117 & PTHq & $\begin{array}{l}\text { Cierre; } \\
24 \text { h pos }\end{array}$ & Calcemia & $1 \mathrm{pmol} / \mathrm{l}$ & $\begin{array}{l}\text { Valor de PTH al cierre tiene }>\text { S, } \\
\text { E y VP que la calcemia y la PTH } \\
\text { a las } 24 \mathrm{~h}\end{array}$ \\
\hline Cahill, et al. ${ }^{4}$ & 2005 & Prospectivo & 31 & PTHq & $\begin{array}{l}\text { Preop; } \\
\text { intra*; } \\
\text { cierre }\end{array}$ & $\begin{array}{l}\text { Calcemia } \\
\text { pre, } 24,48 \\
\text { y } 72 \text { h pos }\end{array}$ & $>50 \%$ & $\begin{array}{l}\text { El uso de PTH como valor } \\
\text { predictivo es relativo, requiere } \\
\text { controles posteriores }\end{array}$ \\
\hline $\begin{array}{l}\text { Lombardi, } \\
\text { et al. }^{5}\end{array}$ & 2006 & Prospectivo & 523 & PTHi & $4 \mathrm{~h}$ pos & $\begin{array}{l}\text { Calcemia } \\
24,48, \text { día } \\
7 \text { pos }\end{array}$ & $<15 \mathrm{pg} / \mathrm{ml}$ & $\begin{array}{l}\text { Valor de PTH a las } 4 \text { h pos no } \\
\text { predice adecuadamente el } \\
\text { hipoparatiroidismo }\end{array}$ \\
\hline $\begin{array}{l}\text { Karatzanis, } \\
\text { et al. }{ }^{7}\end{array}$ & 2017 & Prospectivo & 100 & PTHi & $\begin{array}{l}\text { Preop; } 18 \\
\text { y } 24 \text { h pos }\end{array}$ & $\begin{array}{l}\text { Calcemia, } \\
\text { Mg, Py alb } \\
\text { cada } 12 \mathrm{~h}^{\dagger}\end{array}$ & $56 \%$ & $\begin{array}{l}\text { Valores de PTH al primer día } \\
\text { pos pueden usarse como } \\
\text { predictor de hipocalcemia }\end{array}$ \\
\hline Pisanu, et al..$^{9}$ & 2012 & Prospectivo & 112 & PTHi & $\begin{array}{l}\text { Preop; } 6 \\
24 \text { y } 48 \text { h } \\
\text { pos }\end{array}$ & $\begin{array}{l}\text { Calcemia } \\
\text { y P pre, } 6 \\
24 \text { y } 48 \text { h }\end{array}$ & $\leq 12,1 \mathrm{pg} / \mathrm{ml}$ & $\begin{array}{l}\text { Una sola medición de PTHi } \\
\text { en el mismo día pos }(6 \mathrm{~h}) \\
\text { y calcemia }(24 \text { h) predice } \\
\text { hipocalcemia }\end{array}$ \\
\hline Pattou, et al. ${ }^{11}$ & 1998 & Prospectivo & 1071 & PTHi & & $\begin{array}{l}\text { Calcemia, } \\
\text { P }\end{array}$ & $\leq 12 \mathrm{pg} / \mathrm{ml}$ & $\begin{array}{l}\text { Valores } \leq 12 \mathrm{pg} / \mathrm{ml} \text { de PTH } \\
\text { predicen hipoparatiroidismo } \\
\text { definitivo }\end{array}$ \\
\hline Payne, et al. ${ }^{12}$ & 2005 & Prospectivo & 95 & PTHi & 1 y 6 h pos & $\begin{array}{l}\text { Calcemia } 1 \\
\text { y } 6 \text { h pos }\end{array}$ & $\leq 8 \mathrm{ng} / \mathrm{l}$ & $\begin{array}{l}\text { Valor de PTH y calcemia a } \\
1 \text { y } 6 \text { h del mismo día pos } \\
\text { significó descenso en los costos } \\
\text { hospitalarios }\end{array}$ \\
\hline Graff, et al. ${ }^{13}$ & 2010 & Retrospectivo & 69 & PTHi & Pos & $\begin{array}{l}\text { Calcemia } 6 \\
\text { y } 18 \text { h pos }\end{array}$ & $<14 \mathrm{pg} / \mathrm{ml}$ & $\begin{array}{l}\text { Una sola medición de PTHi } \\
\text { en el mismo día pos predice } \\
\text { hipocalcemia; aumenta } \\
\text { sensibilidad combinado con } \\
\text { calcemia a las } 6 \mathrm{~h}\end{array}$ \\
\hline Sahli, et al. ${ }^{15}$ & 2018 & Prospectivo & 218 & PTHi & $\begin{array}{l}\text { Pre; } 1 \mathrm{~h} \\
\text { pos }\end{array}$ & Calcemia & $\begin{array}{c}<10 \mathrm{y}<20 \mathrm{pg} / \mathrm{ml} \\
\quad \text { desc }>50 \%\end{array}$ & $\begin{array}{l}\text { Los valores de PTH no son } \\
\text { confiables para predecir } \\
\text { hipocalcemia }\end{array}$ \\
\hline Carr, et al. ${ }^{16}$ & 2014 & Retrospectivo & 77 & PTHi & $\begin{array}{l}4 \text { h pos; } \\
\text { día } 1 \text { pos }\end{array}$ & $\begin{array}{l}\text { Calcemia, } \\
\text { vitamina D }\end{array}$ & $<10 \mathrm{pg} / \mathrm{ml}$ & $\begin{array}{l}\text { Una sola medición de PTHi } \\
\text { en el mismo día pos predice } \\
\text { necesidad de reposición oral de } \\
\text { calcio o control de calcemia }(4 \mathrm{~h})\end{array}$ \\
\hline $\begin{array}{l}\text { AES } \\
\text { Guidelines }^{17}\end{array}$ & 2007 & Retrospectivo & 458 & PTHi/q & $\begin{array}{l}\text { Entre } 1 \mathrm{~h} \mathrm{y} \\
18 \mathrm{~h} \text { pos }\end{array}$ & Calcemia & & $\begin{array}{l}\text { Valores normales de PTH } \\
\text { predicen normocalcemia, } \\
\text { preferentemente a las } 4 \mathrm{~h} \text { pos }\end{array}$ \\
\hline $\begin{array}{l}\text { Calvo Espino, } \\
\text { et al. }{ }^{18}\end{array}$ & 2019 & Retrospectivo & 481 & PTHi & $\begin{array}{l}16-20 \mathrm{~h} \\
\text { pos }\end{array}$ & $\begin{array}{l}\text { Calcemia } \\
16-20 \mathrm{~h} \\
\text { pos }\end{array}$ & $<5 \mathrm{pg} / \mathrm{ml}$ & $\begin{array}{l}\text { Valor de PTH } \\
<5 \text { pg/ml primer día pos predice } \\
\text { hipoparatiroidismo definitivo }\end{array}$ \\
\hline $\begin{array}{l}\text { Almquist, } \\
\text { et al. } .^{19}\end{array}$ & 2014 & Prospectivo & 519 & PTHi/q & $\begin{array}{l}\text { Intra; día } 1 \\
\text { y } 14 \text { pos }\end{array}$ & Calcemia & $<6,6 \mathrm{pg} / \mathrm{ml}$ & $\begin{array}{l}\text { Valores bajos de PTH al } \\
\text { primer día pos predice } \\
\text { hipoparatiroidismo definitivo }\end{array}$ \\
\hline $\begin{array}{l}\text { Hermann, } \\
\text { et al. }{ }^{20}\end{array}$ & 2008 & Prospectivo & 208 & PTHi & $\begin{array}{l}\text { Preop; } \\
\text { intra }{ }^{\ddagger} ; 3 \mathrm{~h} \text {; } \\
\text { día 1, 2, } \\
3 \text { pos }\end{array}$ & Calcemia & & $\begin{array}{l}\text { Valores normales de PTH } \\
\text { medidos en el mismo día pos } \\
\text { predicen mejor el riesgo de } \\
\text { hipocalcemia persistente que al } \\
\text { final de la cirugía }(3 \mathrm{~h})\end{array}$ \\
\hline
\end{tabular}


Cirugía y Cirujanos. 2020;88(1)

Tabla 1. Resumen de estudios con sus diseños y principales hallazgos y conclusiones (Continuación)

\begin{tabular}{|c|c|c|c|c|c|c|c|c|}
\hline Autores & Año & Diseño & $\mathrm{n}$ & PTH & $\begin{array}{l}\text { Tiempo } \\
\text { para PTH }\end{array}$ & Control & $\begin{array}{c}\text { Valor de corte } \\
\text { PTH }\end{array}$ & Conclusión \\
\hline Khafif, et al. ${ }^{21}$ & 2006 & Prospectivo & 40 & PTHi & $\begin{array}{l}\text { Preop; } \\
30 \mathrm{~min} \\
\text { pos }\end{array}$ & Calcemia & Desc $>50 \%$ & $\begin{array}{l}\text { Una disminución relativa } \\
\text { del } 50 \% \text { de la PTH predice } \\
\text { hipocalcemia }\end{array}$ \\
\hline $\begin{array}{l}\text { Galy-Bernadoy, } \\
\text { et al. }{ }^{22}\end{array}$ & 2018 & Prospectivo & 257 & PTHi & $\begin{array}{l}\text { Cierre (2 } \\
\text { kits) }\end{array}$ & Calcemia & $<7 \mathrm{y}<4 \mathrm{ng} / \mathrm{l}$ & $\begin{array}{l}\text { Una sola medición de PTHi } \\
\text { al cierre de piel predice } \\
\text { hipoparatiroidismo }\end{array}$ \\
\hline $\begin{array}{l}\text { Grodski y } \\
\text { Farrell }^{23}\end{array}$ & 2007 & Prospectivo & 76 & PTHi & $\begin{array}{l}4-12 \mathrm{~h} \\
\text { pos }\end{array}$ & $\begin{array}{l}\text { Calcemia } \\
24 \mathrm{~h}\end{array}$ & $12 \mathrm{pg} / \mathrm{ml}$ & $\begin{array}{l}\text { Una sola medición de PTHi a las } \\
4-12 \text { h pos predice el riesgo de } \\
\text { hipocalcemia }\end{array}$ \\
\hline Lim, et al. ${ }^{24}$ & 2009 & Prospectivo & 21 & PTHi & $1 \mathrm{~h} \mathrm{pos}$ & Calcemia & $\leq 2,5 \mathrm{pmol} / \mathrm{l}$ & $\begin{array}{l}\text { Una sola medición de PTHi } \\
\text { en el mismo día pos predice } \\
\text { hipocalcemia sintomática ( } 1 \text { h) }\end{array}$ \\
\hline Kim, et al. ${ }^{25}$ & 2011 & Retrospectivo & 112 & PTHi & $1 \mathrm{~h} \mathrm{pos}$ & Calcemia & $<10 \mathrm{pg} / \mathrm{ml}$ & $\begin{array}{l}\text { Una sola medición de PTHi } \\
\text { en el mismo día pos predice } \\
\text { hipocalcemia sintomática } \\
(1 \mathrm{~h}) \text {; aumenta S con calcemia } \\
\text { sérica }\end{array}$ \\
\hline Le, et al. ${ }^{26}$ & 2014 & Retrospectivo & 125 & PTHi & $1 \mathrm{~h} \mathrm{pos}$ & Calcemia & $<12 \mathrm{pg} / \mathrm{ml}$ & $\begin{array}{l}\text { Una sola medición de PTHi en } \\
\text { el mismo día pos estratifica a } \\
\text { los pacientes según riesgo de } \\
\text { hipocalcemia ( } 1 \mathrm{~h} \text { ) }\end{array}$ \\
\hline Kim, et al. ${ }^{27}$ & 2013 & Prospectivo & 108 & PTHi & $6 \mathrm{~h} \mathrm{pos}$ & $\begin{array}{l}\text { Calcemia, } \\
\text { Mg, P }\end{array}$ & $<10,6 \mathrm{mg} / \mathrm{dl}$ & $\begin{array}{l}\text { Una sola medición de PTHi } \\
\text { en el mismo día pos predice } \\
\text { hipocalcemia ( } 6 \mathrm{~h} \text { ) }\end{array}$ \\
\hline Sywak, et al. ${ }^{28}$ & 2007 & Prospectivo & 100 & PTHi & $\begin{array}{l}4 \mathrm{~h} ; 24 \mathrm{~h} \\
\text { pos }\end{array}$ & Calcemia & $\leq 3 \mathrm{ng} / \mathrm{l}$ a las $4 \mathrm{~h}$ & $\begin{array}{l}\text { Una sola medición de PTHi } \\
\text { en el mismo día pos predice } \\
\text { hipocalcemia }(4 \mathrm{~h})\end{array}$ \\
\hline $\begin{array}{l}\text { Pradeep y } \\
\text { Ramalingam }{ }^{30}\end{array}$ & 2014 & Prospectivo & 203 & PTHi & $\begin{array}{l}\text { Preop; } \\
8 \text { h pos }\end{array}$ & $\begin{array}{l}\text { Calcemia } \\
24-48 \text { h y } \\
\text { vitamina D }\end{array}$ & & $\begin{array}{l}\text { El descenso de PTH en } \\
\text { pacientes con déficit de } \\
\text { vitamina D no predice } \\
\text { hipocalcemia }\end{array}$ \\
\hline
\end{tabular}

Alb: albúmina; desc: descenso; E: especificidad; intra: intraoperatorio; Mg: magnesemia; P: fosfatemia; pos: posoperatorio; PTH: parathormona; pre: preoperatorio; PTHi: PTH intacta; PTHq: PTH quick; S: sensibilidad; VP: valor predictivo.

*Posterior a movilización tiroidea/al resecar la glándula.

tHasta tres mediciones consecutivas normales.

${ }^{\ddagger} \mathrm{A}$ los 10 minutos de cada lobectomía.

et al. ${ }^{3}$ también concluyen que una única muestra al finalizar cirugía, durante el cierre de la piel, con el paciente anestesiado, permitiría estratificar el riesgo y evitaría las molestias de la extracción. Ambos estudios justifican con esto que el momento ideal para la toma de la muestra es el intraoperatorio o el posoperatorio inmediato. Galy-Bernadoy, et al. ${ }^{22}$ también toman la muestra al momento del cierre de la piel, pero lo interesante de su estudio es que usaron dos kits para el dosaje de PTH, obteniendo dos valores de corte con cada uno. El objetivo del estudio fue identificar a los pacientes sin riesgo de hipocalcemia con un valor predictivo negativo del $100 \%$. Para este grupo, los valores de corte fueron $\geq 9 \mathrm{y} \geq 19 \mathrm{ng} / \mathrm{l}$, cada uno con un valor predictivo negativo y una sensibilidad del $100 \%$, y una especificidad algo menor. El $14-28 \%$ de los pacientes quedaron en una «zona gris» o intermedia en relación con los valores de corte y fueron tratados en forma preventiva. Por otro lado, en contraposición, Cahill, et al. ${ }^{4}$ afirman que, debido al habitual descenso de la PTH intraoperatoria en la cirugía bilateral de tiroides, un valor bajo intraoperatorio no permite predecir el riesgo de hipocalcemia, por lo que sugieren un dosaje posterior a la cirugía.

Otros grupos realizan la medición en el posoperatorio inmediato o mediato, predominando la toma de muestra entre las 4 y las 6 horas posoperatorias. Khafif, et al. ${ }^{21}$ demostraron la aplicabilidad del método con muestras tomadas 30 minutos después de la 
cirugía. Por su parte, Payne, et al..$^{12}$ demostraron una adecuada sensibilidad de la PTH tomada 1 y 6 horas después de la cirugía. Lombardi, et al. ${ }^{5}$ obtuvieron muestras en diferentes intervalos del posoperatorio, encontrando inicialmente una mayor precisión en los valores obtenidos a las 4-6 horas de la cirugía. En un trabajo posterior, con mayor número de pacientes, concluyen que el valor tomado a las 4 horas no sería lo suficientemente preciso, y que se debería continuar midiendo la calcemia. Por su parte, Carr et al. $^{16}$, con 77 pacientes, obtuvieron buenos resultados con una muestra tomada a las 4 horas. Asimismo, el grupo de Australian Endocrine Surgeons ${ }^{17}$ realizó una revisión retrospectiva que incluyó a 458 pacientes de distintos hospitales; si bien no exponen un valor de corte de la PTH, recomiendan su medición a las 4 horas de la cirugía y señalan que valores normales de PTH predicen la normocalcemia, por lo que los pacientes pueden ser dados de alta en el primer día del posoperatorio ${ }^{16,17}$. En concordancia, Sywak, et al. ${ }^{28}$ también han recomendado el uso de una única muestra tomada a las 4 horas. Grodski y Farrell ${ }^{23}$ concluyeron que los pacientes con valores normales de PTH posquirúrgicos pueden ser dados de alta en forma segura el primer día del posoperatorio. En su trabajo, las mediciones se realizaron a las 4 y 12 horas de la cirugía. Por otro lado, en el trabajo de Kim, et al. ${ }^{27} \mathrm{con}$ 108 pacientes, un valor menor de 10,6 mg/dl a las 6 horas de la cirugía cuenta con una sensibilidad del $89 \%$, una especificidad del $88 \%$ y un valor predictivo positivo del $85 \%$. Calvo Espino, et al. ${ }^{18}$ demostraron que una medición en el primer día del posoperatorio tiene una sensibilidad del $95 \%$ y un valor predictivo negativo del $99,6 \%$ para demostrar hipoparatiroidismo permanente si los valores de PTH se encuentran por debajo de $5 \mathrm{pg} / \mathrm{ml}$. Por ello concluyen que, si bien aún no existen valores absolutos, los obtenidos en su trabajo pueden predecir aquellos pacientes en riesgo debido al alto un valor predictivo negativo obtenido.

Otro punto clave que también genera discrepancia entre los autores es el número de muestras. Si bien muchos sugieren una sola muestra, otros prefieren repetir el análisis a intervalos variables, por ejemplo cada 1, 6 y 12 horas, por lo que no puede hacerse una extrapolación para un uso sistemático. Como se ha mencionado antes, la corta vida media de la hormona explica que la meseta de PTH se alcance durante los primeros minutos del posoperatorio ${ }^{24,27}$. Como consecuencia de esto, muchos sostienen que las mediciones seriadas de PTH no estarían justificadas, ya que no existirían variaciones significativas entre cada valor. Además, la evidencia actual permite aseverar que una sola muestra de PTH cuenta con una sensibilidad y una especificidad adecuadas, independientemente del momento de la toma ${ }^{7,8,10,29}$.

La interpretación del valor de PTH también es motivo de controversia. Algunos autores prefieren utilizar un valor absoluto de PTH, por ejemplo $5 \mathrm{pg} / \mathrm{ml}^{18}$, $10 \mathrm{pg} / \mathrm{ml}^{9}$, $8 \mathrm{mg} / \mathrm{dl}^{1}$ y $1 \mathrm{pmol} / /^{3}$, mientras que otros consideran que la mayor precisión se alcanza comparando valores preoperatorios, intraoperatorios y posoperatorios, es decir, valores relativos. Khafif, et al. ${ }^{21}$, en su estudio prospectivo con 40 pacientes, demostraron una sensibilidad del $92 \%$ y una especificidad del $66 \%$ cuando hay un descenso del $50 \%$ entre los valores de PTH preoperatoria y los determinados a los 30 minutos; del $23 \%$ y el $75 \%$, respectivamente, cuando el descenso de la PTH es del $75 \%$ a igual intervalo; y del $46 \%$ y el $100 \%$, respectivamente, tomando un valor absoluto como límite (normalidad). Lógicamente, a menor valor de corte de PTH establecido, mayor es la especificidad y menor es la sensibilidad. Karatzanis, et al. ${ }^{7}$, en forma prospectiva enrolando 100 pacientes, utilizaron valores relativos, indicando calcio al alta o prolongando la internación cuando el descenso era mayor del $56 \%$ y el $62 \%$, respectivamente. En relación con la evidencia actual, se puede concluir que tanto el valor absoluto como un descenso relativo permitirían predecir el hipoparatiroidismo posoperatorio y el riesgo de hipocalcemia. Sin embargo, el análisis relativo obliga a contar con un valor basal previo a la cirugía.

Algunos autores han asociado el análisis de la PTH con el de la calcemia para aumentar la eficacia predictiva. Pisanu, et al. ${ }^{9}$ relacionaron un valor de corte absoluto de PTH de $12 \mathrm{pg} / \mathrm{dl}$ o un descenso igual o mayor del $70,5 \%$ en la PTH preoperatoria y posoperatoria (a las $6 \mathrm{~h}$ ) con un valor de corte de la calcemia de $7,97 \mathrm{mg} / \mathrm{dl}$ tomado a las 24 horas, y demostraron una sensibilidad y una especificidad de $100 \%$ para predecir la hipocalcemia. Asimismo, Graff, et al..$^{13}$, en forma prospectiva, también demostraron un aumento de la especificidad del 83 al $88 \%$ para detectar la hipocalcemia al combinar los valores de la PTH (14 pg/dl) con los de la calcemia $(8,0 \mathrm{mg} / \mathrm{dl})$, tomadas a las 6 horas de la cirugía. De similar manera, Kim, et al. ${ }^{25}$ evidenciaron mayores sensibilidad y especificidad combinando la PTH y la calcemia. Ciertamente, estas asociaciones implican un mayor costo económico y en muchos casos una demora en la externación (calcemia tomada a las $24 \mathrm{~h}$ ), pero evidentemente se asociarían a un aumento de la capacidad predictiva. 
En cuanto a la aplicabilidad del uso de la PTH como predictor clínico, algunos estudios ponen en duda su empleo en pacientes con déficit de vitamina $D$ y en aquellos con enfermedad de Graves. En su estudio, Pradeep y Ramalingam ${ }^{30}$ analizaron la utilidad de la PTH tomada a las 8 horas de la cirugía y encontraron una menor sensibilidad para predecir la hipocalcemia en los pacientes con déficit preoperatorio de vitamina D. Según los autores, esto se justificaría porque los pacientes con dicho déficit presentan un descenso posoperatorio más tardío de la PTH y valores absolutos de PTH preoperatoria y posoperatoria más elevados. Para estos pacientes sugieren una suplementación preoperatoria de vitamina $\mathrm{D}$ y una toma más tardía de la PTH, entre las 12 y 24 horas. En cuanto al bocio difuso tóxico, Annerbo, et al. ${ }^{31}$ analizaron las variaciones de PTH en pacientes sometidos a tiroidectomía por enfermedad de Graves. En ellos, el monitoreo con PTH no sería recomendable, ya que, a pesar de contar con valores normales de esta hormona, un porcentaje no menor requirió suplementación de calcio o de vitamina $\mathrm{D}$, o de ambos.

\section{Conclusiones}

El hipoparatiroidismo posoperatorio es una complicación frecuente y potencialmente grave de la tiroidectomía total. Se asocia a un importante aumento de los costos de salud.

La necesidad de contar con un predictor adecuado ha impulsado numerosos estudios sobre el uso de la PTH. Si bien el método carece de un 100\% sensibilidad, la PTH es casi sin dudas, hoy en día, un predictor de uso creciente y seguro. La evidencia actual apoya su utilización para estratificar a los pacientes, solo o acompañado de otros predictores, pero aún persisten interrogantes acerca de la mejor manera de implementar su uso. La mayoría de los estudios presenta buenos resultados durante las primeras 4, 6 y 24 horas luego de la cirugía. Asimismo, el reemplazo del dosaje de la calcemia seriada beneficiaría a los pacientes y a los centros asistenciales, al disminuir costos de procesamiento, el tiempo de internación y la medicación suplementaria.

La heterogeneidad de los estudios publicados en relación con los intervalos de toma de la muestra, el valor de corte, las variables medidas, etc., atenta contra la realización de revisiones sistemáticas. Sin embargo, por otro lado, esto evidencia la versatilidad del método, aun con discrepancias en su logística de implementación.
Actualmente, un número creciente de protocolos incluyen la PTH. El estudio prospectivo de los mismos probablemente permitirá contestar en un futuro las preguntas que hoy no tienen una clara respuesta.

\section{Conflicto de intereses}

Los autores declaran no tener conflicto de intereses.

\section{Financiamiento}

Este trabajo no recibió financiamiento para su desarrollo.

\section{Responsabilidades éticas}

Protección de personas y animales. Los autores declaran que para esta investigación no se han realizado experimentos en seres humanos ni en animales.

Confidencialidad de los datos. Los autores declaran que han seguido los protocolos de su centro de trabajo sobre la publicación de datos de pacientes.

Derecho a la privacidad y consentimiento informado. Los autores declaran que en este artículo no aparecen datos de pacientes.

\section{Bibliografía}

1. Quiros RM, Pesce CE, Wilhelm SM, Djuricin G, Prinz RA. Intraoperative parathyroid hormone levels in thyroid surgery are predictive of postoperative hypoparathyroidism and need for vitamin $D$ supplementation. Am J Surg. 2005;189:306-9.

2. Mundy GR, Guise TA. Hormonal control of calcium homeostasis. Clin Chem. 1999;45(8 Pt 2):1347-52.

3. Lang BH, Yih PC, Ng KK. A prospective evaluation of quick intraoperative parathyroid hormone assay at the time of skin closure in predicting clinically relevant hypocalcemia after thyroidectomy. World J Surg. 2012;36:1300-6

4. Cahill RA, Harty R, Cotter S, Watson RG. Parathormone response to thyroid surgery. Am J Surg. 2006;191:453-9.

5. Lombardi CP, Raffaelli M, Princi P, Dobrinja C, Carrozza C, Di Stasio E, et al. Parathyroid hormone levels 4 hours after surgery do not accurately predict post-thyroidectomy hypocalcemia. Surgery. 2006;140:1016-23.

6. Rosato L, Avenia N, Bernante P, De Palma M, Gulino G, Nasi PG, et al. Complications of thyroid surgery: analysis of a multicentric study on 14,934 patients operated on in Italy over 5 years. World J Surg. 2004;28:271-6.

7. Karatzanis AD, lerodiakonou DP, Fountakis ES, Velegrakis SG, Doulaptsi MV, Prokopakis EP. Postoperative day 1 levels of parathyroid as predictor of occurrence and severity of hypocalcaemia after total thyroidectomy. Head Neck. 2018;40:1040-45.

8. Edafe O, Antakia R, Laskar N, Uttley L, Balasubramanian SP. Systematic review and meta-analysis of predictors of post-thyroidectomy hypocalcaemia. Br J Surg. 2014;101:307-20.

9. Pisanu A, Saba A, Coghe F, Uccheddu A. Early prediction of hypocalcemia following total thyroidectomy using combined intact parathyroid hormone and serum calcium measurement. Langenbecks Arch Surg. 2013;398:423-30.

10. Grodski S, Serpell J. Evidence for the role of perioperative PTH measurement after total thyroidectomy as a predictor of hypocalcemia. World J Surg. 2008;32:1367-73.

11. Pattou F, Combemale F, Fabre S, Carnaille B, Decoulx M, Wemeau JL, et al. Hypocalcemia following thyroid surgery: incidence and prediction of outcome. World J Surg. 1998;22:718-24. 
12. Payne RJ, Tewfik MA, Hier MP, Tamilia M, Mac Namara E, Young J, et al. Benefits resulting from 1- and 6-hour parathyroid hormone and calcium levels after thyroidectomy. Otolaryngol Head Neck Surg. 2005;133:386-90.

13. Graff AT, Miller FR, Roehm CE, Prihoda TJ. Predicting hypocalcemia after total thyroidectomy: parathyroid hormone level vs. serial calcium levels. Ear Nose Throat J. 2010;89:462-5.

14. Dionigi G, Bacuzzi A, Bertocchi V, Carrafiello G, Boni L, Rovera F, et al. Prospectives and surgical usefulness of perioperative parathyroid hormone assay in thyroid surgery. Expert Rev Med Devices. 2008;5:699-704.

15. Sahli Z, Najafian A, Kahan S, Schneider E, Zeiger M, Mathur A. One-hour postoperative parathyroid hormone levels do not reliably predict hypocalcemia after thyroidectomy. World J Surg. 2018;42:2128-33.

16. Carr AA, Yen TW, Fareau GG, Cayo AK, Misustin SM, Evans DB, et al A single parathyroid hormone level obtained 4 hours after total thyroidectomy predicts the need for postoperative calcium supplementation. J Am Coll Surg. 2014;219:757-64.

17. AES Guidelines $06 / 01$ Group. Postoperative parathyroid hormone measurement and early discharge after total thyroidectomy: analysis of Australian data and management recommendations. ANZ J Surg. 2007;77:199-202.

18. Calvo Espino PC, Bautista JAR, Caselles MA, González JS, Pavía AG García-Oria MJ, et al. Serum levels of intact parathyroid hormone on the first day after total thyroidectomy as predictor of permanent hypoparathyroidism. Endocrinol Diabetes Nutr. 2019;66:195-201.

19. Almquist $M$, Hallgrimsson $P$, Nordenström $E$, Bergenfelz A. Prediction of permanent hypoparathyroidism after total thyroidectomy. World J Surg. 2014;38:2613-20.

20. Hermann M, Ott J, Promberger R, Kober F, Karik M, Freissmuth M. Kinetics of serum parathyroid hormone during and after thyroid surgery. $\mathrm{Br}$ J Surg. 2008;95:1480-7.

21. Khafif A, Pivoarov A, Medina JE, Avergel A, Gil Z, Fliss DM. Parathyroid hormone: a sensitive predictor of hypocalcemia following total thyroidectomy. Otolaryngol Head Neck Surg. 2006;134:907-10.
22. Galy-Bernadoy C, Lallemant B, Chambon G, Pham HT, Reynaud C, Alovisetti $\mathrm{C}$, et al. Parathyroid hormone assays following total thyroidectomy: is there a predictive value? Eur Thyroid J. 2018;7:34-8.

23. Grodski S, Farrell S. Early postoperative PTH levels as a predictor of hypocalcaemia and facilitating safe early discharge after total thyroidectomy. Asian J Surg. 2007;30:178-82.

24. Lim JP, Irvine R, Bugis S, Holmes D, Wiseman SM. Intact parathyroid hormone measurement 1 hour after thyroid surgery identifies individuals at high risk for the development of symptomatic hypocalcemia. Am J Surg. 2009;197:648-53.

25. Kim JH, Chung MK, Son YI. Reliable early prediction for different types of post-thyroidectomy hypocalcemia. Clin Exp Otorhinolaryngol. 2011;4:95-100.

26. Le TN, Kerr PD, Sutherland DE, Lambert P. Validation of 1-hour post-thyroidectomy parathyroid hormone level in predicting hypocalcemia. J Otolaryngol Head Neck Surg. 2014;43:5.

27. Kim JP, Park JJ, Son HY, Kim RB, Kim HY, Woo SH. Effectiveness of an i-PTH measurement in predicting post thyroidectomy hypocalcemia: prospective controlled study. Yonsei Med J. 2013;54:637-42.

28. Sywak MS, Palazzo FF, Yeh M, Wilkinson M, Snook K, Sidhu SB, et al. Parathyroid hormone assay predicts hypocalcaemia after total thyroidectomy. ANZ J Surg. 2007;77:667-70.

29. Lee DR, Hinson AM, Siegel ER, Steelman SC, Bodenner DL, Stack BC Jr. Comparison of intraoperative versus postoperative parathyroid hormone levels to predict hypocalcemia earlier after total thyroidectomy. Otolaryngol Head Neck Surg. 2015;153:343-9.

30. Pradeep PV, Ramalingam K. Postoperative PTH measurement is not a reliable predictor for hypocalcemia after total thyroidectomy in vitamin D deficiency: prospective study of 203 cases. World J Surg. 2014; 38:564-7.

31. Annerbo M, Hultin $H$, Stalberg P, Hellman P. Left-shifted relation between calcium and parathyroid hormone in graves' disease. J Clin Endocrinol Metab. 2014;99:545-51. 